\title{
The Effects of RF Sputtering Power and Gas Pressure on Structural and Electrical Properties of ITiO Thin Film
}

\author{
Accarat Chaoumead, Youl-moon Sung, and Dong-Joo Kwak \\ Department of Electrical Engineering, Kyungsung University, Busan 608-736, Republic of Korea \\ Correspondence should be addressed to Dong-Joo Kwak, djkwak@ks.ac.kr
}

Received 17 August 2012; Accepted 18 September 2012

Academic Editor: Edvard Kokanyan

Copyright ( $) 2012$ Accarat Chaoumead et al. This is an open access article distributed under the Creative Commons Attribution License, which permits unrestricted use, distribution, and reproduction in any medium, provided the original work is properly cited.

\begin{abstract}
Transparent conductive titanium-doped indium oxide (ITiO) films were deposited on corning glass substrates by RF magnetron sputtering method. The effects of RF sputtering power and Ar gas pressure on the structural and electrical properties of the films were investigated experimentally, using a $2.5 \mathrm{wt} \% \mathrm{TiO}_{2}$-doped $\mathrm{In}_{2} \mathrm{O}_{3}$ target. The deposition rate was in the range of around $20 \sim 60 \mathrm{~nm} / \mathrm{min}$ under the experimental conditions of $5 \sim 20$ mTorr of gas pressure and $220 \sim 350 \mathrm{~W}$ of RF power. The lowest volume resistivity of $1.2 \times 10^{-4} \Omega$-cm and the average optical transmittance of $75 \%$ were obtained for the ITiO film, prepared at $\mathrm{RF}$ power of $300 \mathrm{~W}$ and Ar gas pressure of 15 mTorr. This volume resistivity of $1.2 \times 10^{-4} \Omega$-cm is low enough as a transparent conducting layer in various electrooptical devices, and it is comparable with that of ITO or $\mathrm{ZnO}: \mathrm{Al}$ conducting layer.
\end{abstract}

\section{Introduction}

In the last decade transparent conducting thin films have attracted much attention due to their wide range of applications in electrooptical devices. High-quality transparent conducting films are characterized by a high transmittance in the optical range from 400 to $800 \mathrm{~nm}$ and by an electrical resistivity as low as possible. Moreover, the increases in use of transparent electrodes for many optical devices such as solar cell, PDP, and heat mirrors, so forth, have accelerated the development of inexpensive transparent materials. Indiumtinoxide (ITO) and aluminum-doped zinc oxide ( $\mathrm{ZnO}: \mathrm{Al}$ ) thin films have been widely used as a transparent conducting electrode of various electrooptical devices. Fdoped tinoxide (FTO) has been also well used, especially in a dye-sensitized solar cell (DSC). However, the FTO/ITO films, which are prepared by the chemical vapor deposition (CVD) method, are costly and complicated for mass production. The FTO/ITO films have also limitations in their infrared ray (IR) transmission and thermal resistance as a transparent conductor.

Titanium-doped indiumoxide (ITiO) has also emerged as a transparent conducting oxide (TCO) because it has a low sheet resistance, aside from its high mobility and nearIR transmittance. The properties of ITiO layer show that the long-wavelength falloff in transmittance does not occur until $1500 \mathrm{~nm}$ compared to about $1000 \mathrm{~nm}$ for an $8 \Omega / \mathrm{sq}$ sheet resistivity [1]. Usually, DSCs are still responsive in the 1000$1100 \mathrm{~nm}$ range. Thus, they benefit from the improved TCO layer transmission in this near-IR wavelength range [2]. Therefore, the electrical and optical properties of ITiO thin film should be studied in order to investigate the possible application of the film as a transparent conducting electrode for DSCs and other electrooptical devices. However, only a few papers on the growth of the ITiO thin film were studied systematically and published [3-5].

In this paper, in order to develop a good quality of TCO with low resistivity and high optical transmittance, ITiO thin films are deposited on glass substrates by RF magnetron sputtering, which has many advantages, such as high density and low-temperature plasma production at low gas pressures, using a $2.5 \mathrm{wt} \% \mathrm{TiO}_{2}$-doped $\mathrm{In}_{2} \mathrm{O}_{3}$ target. The influences of RF sputtering power and Ar gas pressure on the structural and electrical properties were mainly studied, and the conduction mechanism of ITiO thin film was discussed.

\section{Experimental}

ITiO films were deposited onto corning glasses by using RF $(13.56 \mathrm{MHz})$ magnetron sputtering method. The ITiO 
target is composed of $97.5 \mathrm{wt} \% \mathrm{In}_{2} \mathrm{O}_{3}$, and $2.5 \mathrm{wt} \% \mathrm{TiO}_{2}$ with a purity of $99.99 \%$. The chamber was of stainless steel of internal diameter $375 \mathrm{~mm}$. The target diameter was $80 \mathrm{~mm}$, and the distance from the target to the substrate was $60 \mathrm{~mm}$. A temperature controller and a heater controlled the substrate temperature. Cooling water was circulated through the target and the chamber to prevent overheating during the deposition. The chamber is exhausted at $1 \times 10^{-5}$ Torr or lower. The gas pressure was in the range of 5 20 mTorr. The samples were supplied at different RF powers, ranging from 220 to $350 \mathrm{~W}$ for 50 minutes.

The resistivity was measured by the four-point probe (Dasol Eng., FPP-HS8) and the hall-effect measurements (ECOPIA HMS-2000). The transmittance of the films was measured with an UV spectrophotometer (Hitachi Co., U3000 ) in the range of $300-800 \mathrm{~nm}$. The crystallographic orientation of the films was determined by an X-ray diffractometer (Rigaku Co., D/max $2100 \mathrm{H}$ ), using $\mathrm{Cu} \mathrm{K \alpha}$ irradiation $(\lambda=1.5418 \AA)$. The morphology of the film was investigated by the atomic force microscopy (AFM). The particle morphology and size were also investigated by the field emission scanning electron microscope (FE-SEM).

\section{Results}

According to our previous studies $[6,7]$, the morphological and the electrical properties of the ITiO thin film were greatly influenced by Ti doping concentration and substrate temperature. The lowest electrical resistivity was obtained at around $400^{\circ} \mathrm{C}$ of substrate temperature and $2.5 \mathrm{wt} \%$ of doping concentration. Therefore all the films in this study were deposited at these conditions.

3.1. Structural Properties. In this paper, the effects of RF power and Ar gas pressure on the X-ray diffraction patterns of ITiO films are studied to optimize the deposition conditions of Ar gas pressure and RF power. The results are shown in Figures 1 and 2, respectively. In Figure 1 all the patterns are those at different discharge powers from 220 to $350 \mathrm{~W}$ and at $15 \mathrm{mTorr}$ of Ar gas pressure. According to Figure 1, the ITiO thin films produced strong (222) peaks of a preferred orientation regardless of sputtering powers and they are located at $2 \theta=30.7^{\circ}$, which is very close to that of the standard $\operatorname{In}_{2} \mathrm{O}_{3}$ crystal $\left(2 \theta=30.61^{\circ}\right)$. The very weak peaks of (400) and (440) crystal planes were also observed, indicating a polycrystalline structure; however, they cannot be comparable to those of (222) preferred orientation. All the peak positions of (222) plane as well as (400) and (440) planes were found to be in good agreement with crystalline indium oxide, and no extra peaks due to the addition of titanium in the indium oxide films were observed.

It is notable that the (222) peaks become more intense and sharper as the sputtering power increases to $300 \mathrm{~W}$ and the intensity of the peak decreases for higher sputtering power. This means that the crystallinity of the films is improved and the grain size becomes larger with increasing sputtering power to $300 \mathrm{~W}$. This result can be explained by the following reasons. As well known from the previous papers the numbers or energy of electrons and ions in
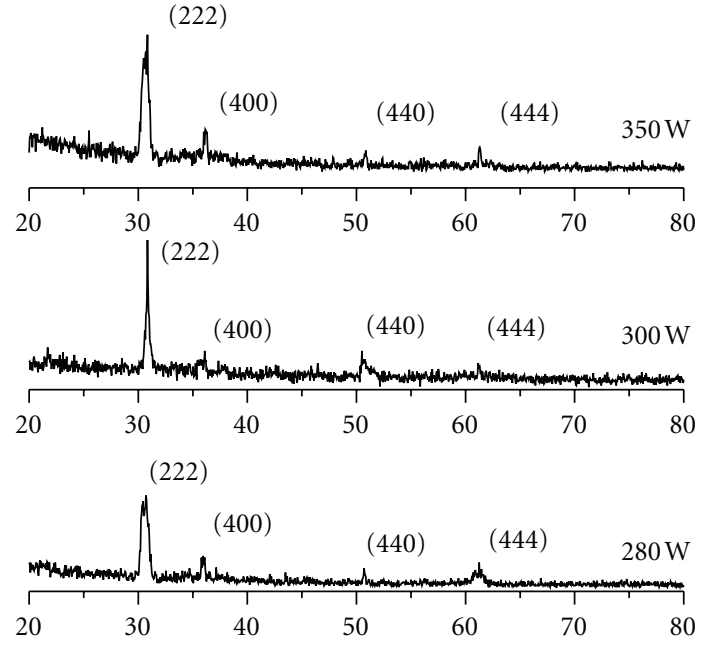

(222)

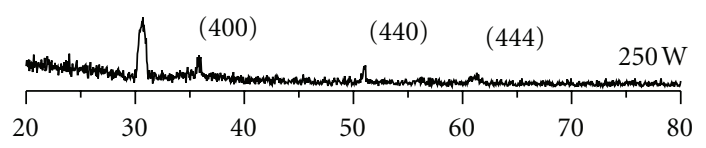

(222)

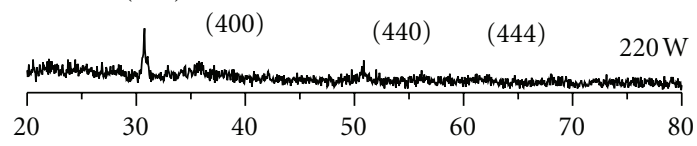

FIGURE 1: XRD patterns of the ITiO films deposited at different RF powers.

glow discharge plasma will increase with an increment in sputtering power, in the case of using metal oxide target $[8,9]$. That is, the energy of sputtered atom which is obtained from the positive ions colliding to target will increase with increasing sputtering power. Thus, the energy of the sputtered atom arriving at the substrate increases with increasing sputtering power to $300 \mathrm{~W}$ and facilitates further crystallization, resulting in the strong (222) preferred orientation. It is also possible that the highly energized electrons with increasing sputtering power will bombard the surface of growing film at the substrate, providing in the form of thermal energy. This energy might act as an additional energy to promote the growth of crystalline. Therefore, it can be concluded that as the sputtering power increases, the number and the momentum of the sputtering particles will also increase. The atoms become more mobile on the surface of the film as the result of the higher bombardment rates on them.

In Figure 2, which shows the results at different gas pressures and at $300 \mathrm{~W}$ of RF power, the intensity of XRD peaks clearly increases with increasing gas pressure up to $15 \mathrm{mTorr}$ and decreases again for higher gas pressure. This result can be explained by the following reasons. As the gas pressure decreases, the mean free path of electron increases. Electrons having the longer mean free path can store higher energy before collisions with Ar gas molecules, and they can produce active ionizations by collision. It follows that many 

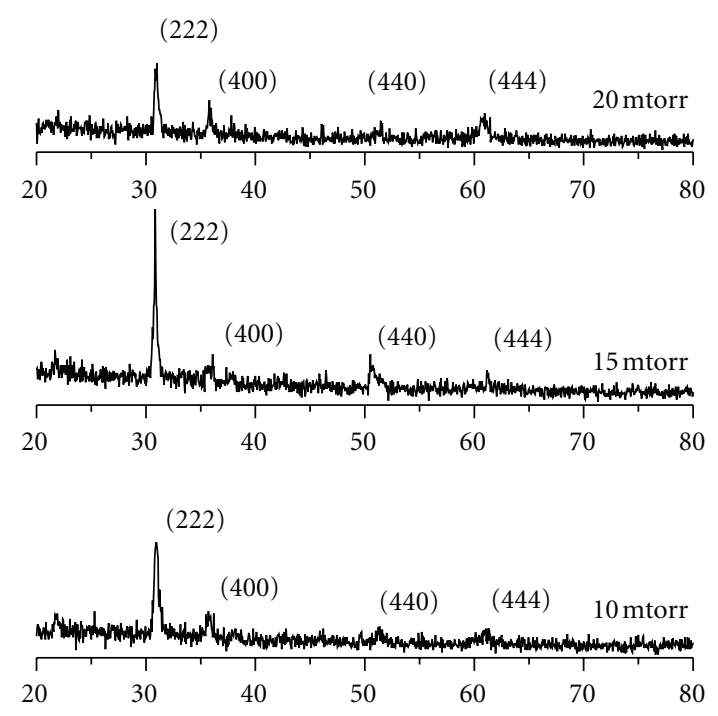

(222)

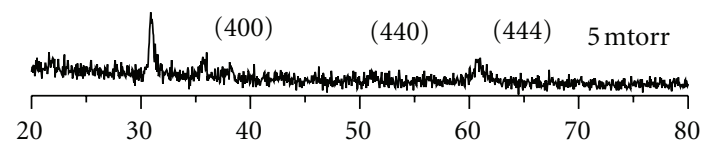

FIGURE 2: XRD patterns of the ITiO films deposited at different gas pressures.

positive ions with higher energy can collide to the target and can sputter more ITiO molecules from the target, giving an increment in deposition rate and an improvement in thin film quality. This result could be also related to an increase in energy of sputtered atom arriving at the substrate because the decrease in gas pressure makes a mean free path of the sputtered atom long. However, when the gas pressure is lower than 15 mTorr, electrons have finite chances of collision with Ar gas atoms and ionization. In the present study, the most effective gas pressure for generation of glow discharge plasma to permit an effective ionization and sputtering is considered as 15 mTorr.

The deposition rate of ITiO film was obtained around $20 \mathrm{~nm} / \mathrm{min}$ to $59.9 \mathrm{~nm} / \mathrm{min}$ in the present experimental conditions of RF sputtering power and gas pressure, and it was also increased with increasing RF power to $300 \mathrm{~W}$ and gas pressure to $15 \mathrm{~m}$ Torr as shown in Figure 3. At these conditions of RF power and gas pressure, the number of ITiO atoms generated from the target by sputtering and the possibility of the ITiO atoms arriving on the substrate without hindrance by collisions increase. The highest deposition rate was $59.9 \mathrm{~nm} / \mathrm{min}$, and it was obtained at the conditions of $300 \mathrm{~W}$ and $15 \mathrm{mTorr}$ of RF power and gas pressure, respectively.

In order to confirm the effect of RF power on the structural properties of the film, the AFM morphologies of the films deposited at different RF power at 15 mTorr of gas pressure were investigated. The results are shown in Figure 4. As the RF power increases up to $300 \mathrm{~W}$, the crystallinity of the film is improved and the crystalline size becomes larger. That
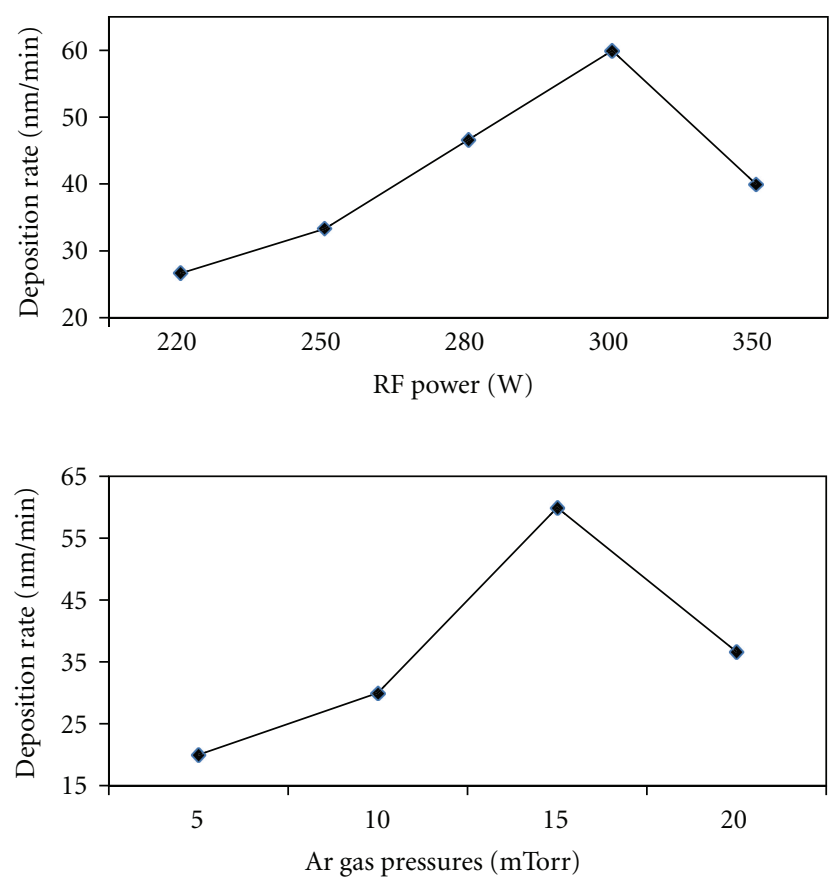

Figure 3: Deposition rate of ITiO film at various RF powers.

is, the increment in sputtering power promotes the growth of crystalline and induces an improvement in crystallinity of the film. However, the excessive supply of RF power over than $300 \mathrm{~W}$ may cause a degradation of the preferred orientation and limits the growth of crystalline grain, resulting in the high defect density and small grain size. The film will suffer from the bombardment of highly energized particles, resulting in internal defects of the film. The crystalline size was estimated by using the Scherrer formula [10]. They were in the ranges of around 2.6 to $13.4 \mathrm{~nm}$ with variation of sputtering power. The largest crystalline size was $13.4 \mathrm{~nm}$, and it was obtained under the conditions of $300 \mathrm{~W}$ of RF power and $15 \mathrm{mTorr}$ of gas pressure. Figure 5 shows the AFM morphology of ITiO film deposited at different gas pressures. The surface morphology was also strongly affected by the gas pressure. The films gradually crystallized with increasing gas pressure up to $15 \mathrm{mTorr}$, and the RMS roughness of the films was lower to $5.9 \mathrm{~nm}$.

3.2. Electrical Properties. Figure 6 indicates the dependence of electrical resistivity of the ITiO films sputtered at $15 \mathrm{mTorr}$ of gas pressure on RF power. It is clear from the figure that the electrical resistivity decreases with increasing sputtering power to $300 \mathrm{~W}$ and thereafter increases slightly. The lowest resistivity is $1.2 \times 10^{-4} \Omega-\mathrm{cm}$, which is low enough as TCO, and it is comparable with that of ITO or ZnO:Al thin film. For the analysis of the conduction mechanism, the hall mobility and the carrier concentration were measured. The results are illustrated in Figure 7. The hall mobility increases with the increase in sputtering power up to $300 \mathrm{~W}$, reaches the maximum value of around $45.5 \mathrm{~cm}^{2} / \mathrm{V} \cdot \mathrm{s}$ at $300 \mathrm{~W}$ of $\mathrm{RF}$ power, and thereafter decreases to $42.7 \mathrm{~cm}^{2} / \mathrm{V} \cdot \mathrm{s}$ at $350 \mathrm{~W}$. 


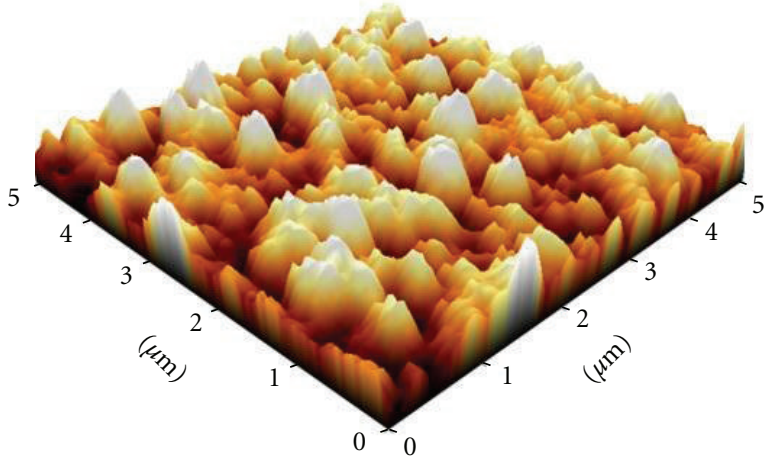

(a)

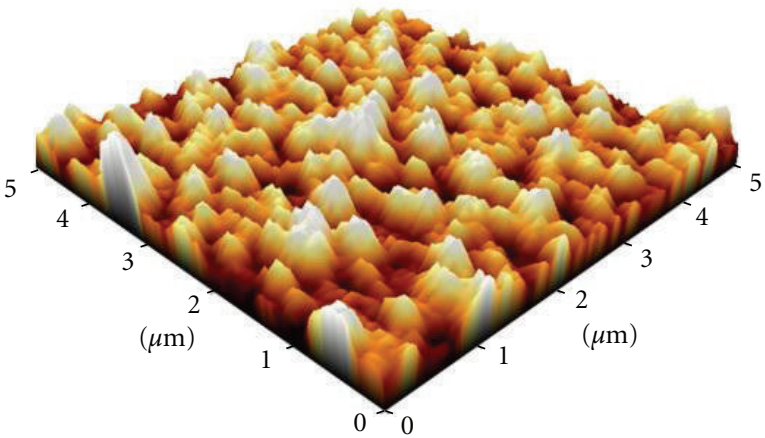

(c)

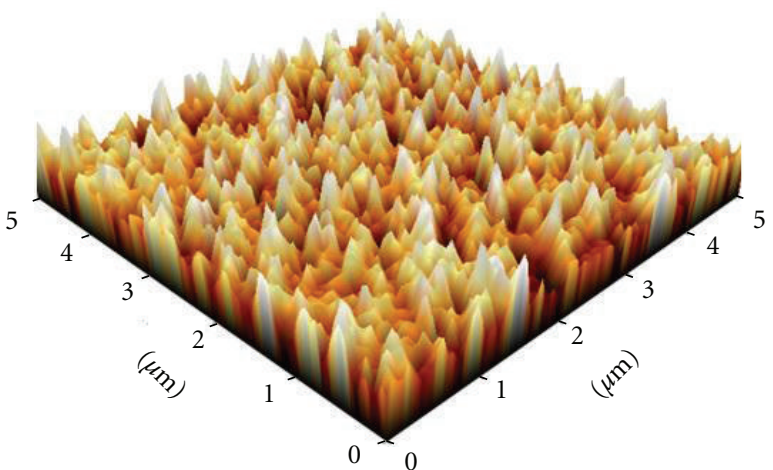

(b)

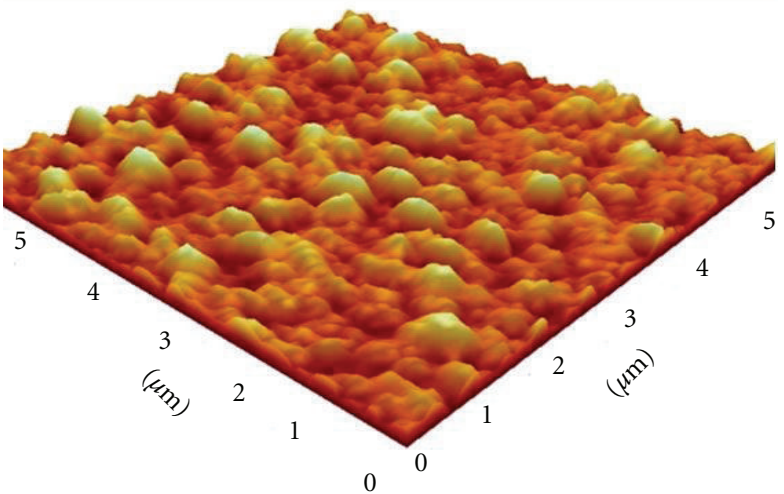

(d)

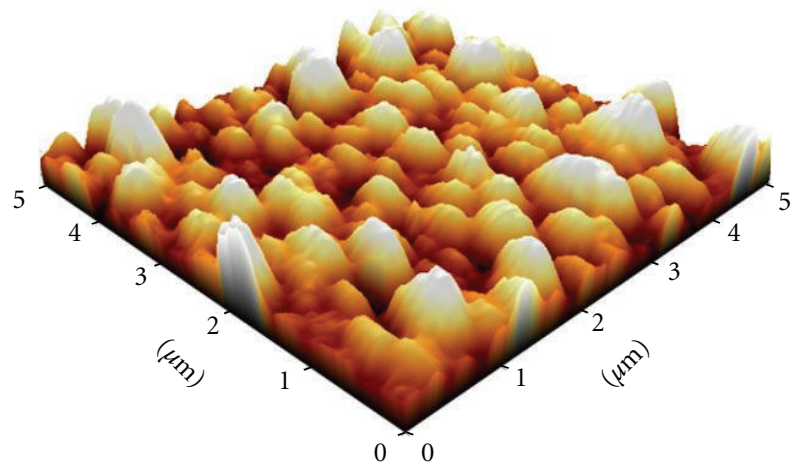

(e)

FIgURE 4: AFM morphologies of the ITiO film at different RF powers. (a) $220 \mathrm{~W}$, (b) $250 \mathrm{~W}$, (c) $280 \mathrm{~W}$, (d) $300 \mathrm{~W}$, (e) $350 \mathrm{~W}$.

A similar trend is observed in the carrier concentration which increases from $6.9 E+20$ to $1.2 E+21 \mathrm{~cm}^{-3}$ as the sputtering power increases from 220 to $300 \mathrm{~W}$. It decreases to $7.7 \mathrm{E}+$ $20 \mathrm{~cm}^{-3}$ thereafter, as also shown in the same figure. In the case of titanium-doped indium oxide, the variation in grain size at the different sputtering powers is considered as one of the causes which affect the conduction mechanism. In our experiment, as mentioned earlier in Figure 1, the XRD peaks become more intense and sharper with increasing sputtering power. This implies that the grain size increases and the grain boundary decreases with increasing in RF power to $300 \mathrm{~W}$. This effect reduces charge carriers scattering due to decrease in grain boundaries, thus increasing the hall mobility. Therefore, the increment in the hall mobility with increasing RF power is ascribed to a decrease in the number of scattering centres for carriers, giving a low electrical resistivity. However, an excessive supply of the sputtering power over $300 \mathrm{~W}$ caused a degradation of the (222) preferred orientation, resulting in high defect density and small grain size. The decrease in grain size increases the grain boundary scattering, thus decreasing the hall mobility and carrier concentration, which causes the poor electrical conductivity. Based on these results, the optimal condition of RF power is $300 \mathrm{~W}$ in this study. 


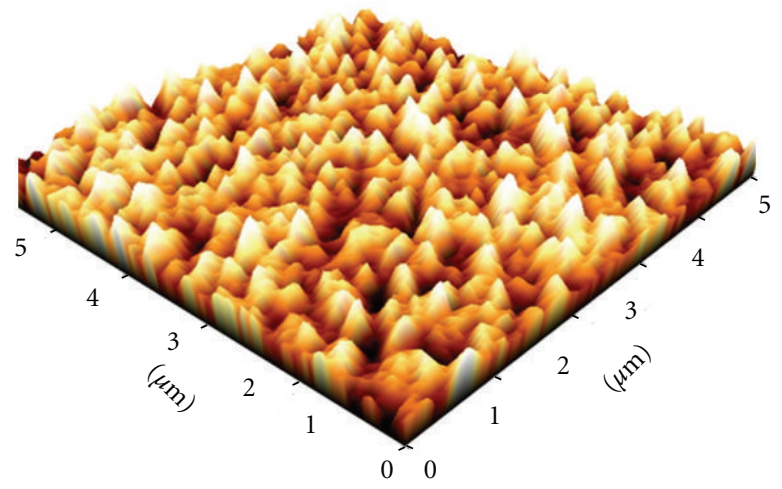

(a)

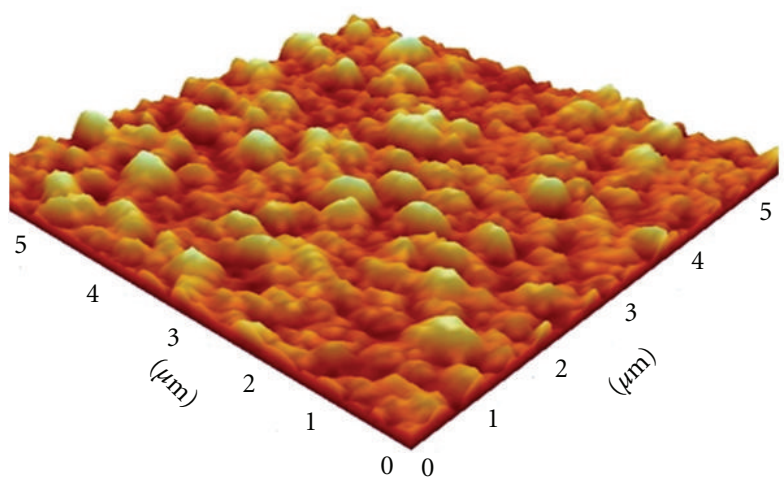

(c)

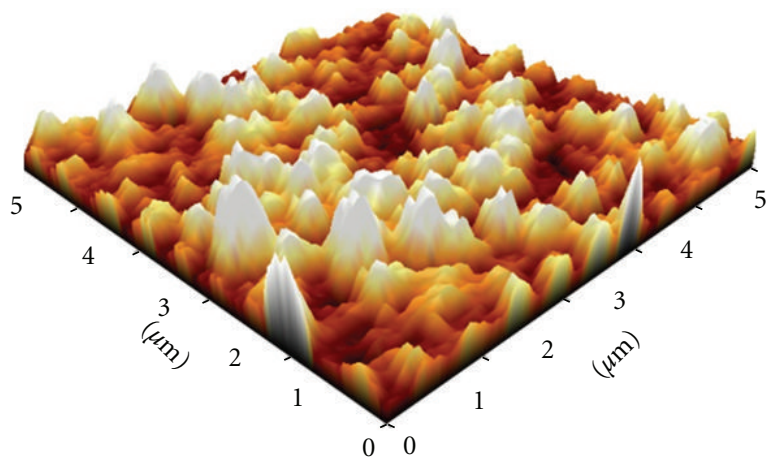

(b)

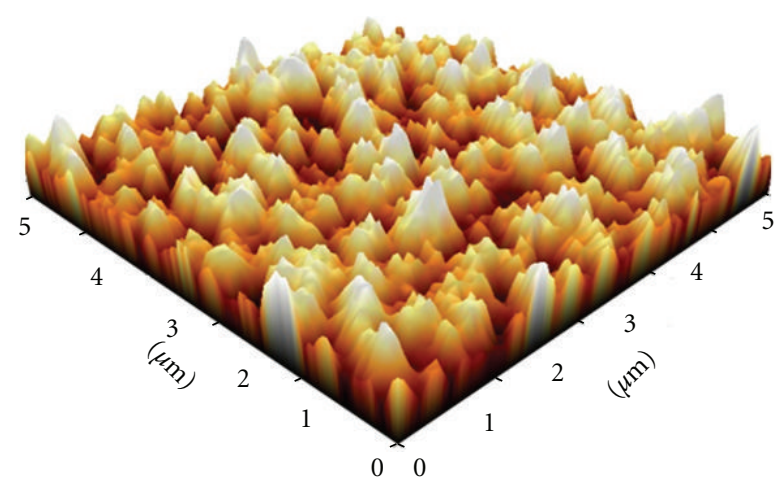

(d)

FIgURE 5: AFM morphologies of the ITiO film at different Ar gas pressures. (a) 5 mTorr, (b) 10 mTorr, (c) 15 mTorr, (d) 20 mTorr.

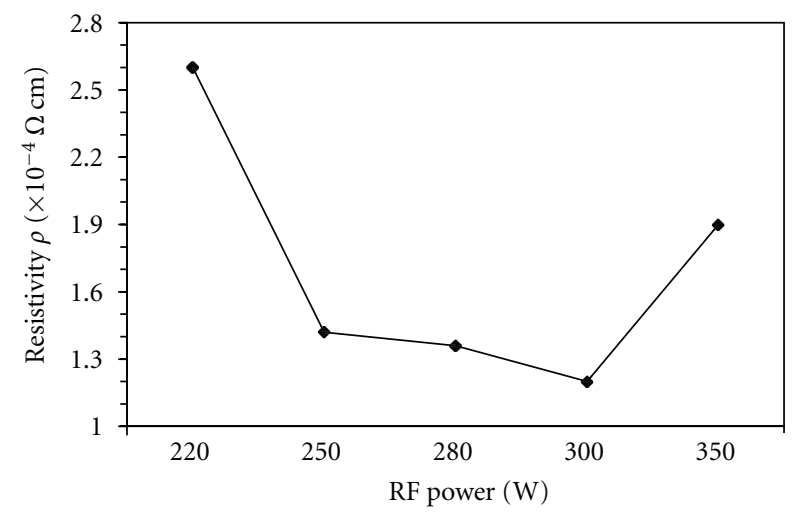

FIGURE 6: Resistivity of the ITiO film with variations of RF power.

Figure 8 illustrates the effect of Ar gas pressure on the electrical resistivity of the ITiO film. The electrical resistivity of the ITiO film decreases form $1.59 \times 10^{-4} \Omega$-cm to $1.2 \times$ $10^{-4} \Omega-\mathrm{cm}$ as the gas pressure increases from 5 to $15 \mathrm{mTorr}$, and the electrical resistivity increases to $1.45 \times 10^{-4} \Omega-\mathrm{cm}$ thereafter, at the higher Ar gas pressure. The hall mobility and the carrier concentration dependencies of ITiO thin film on gas pressure are also measured and presented in Figure 9. The hall mobility was around $35 \mathrm{~cm}^{2} / \mathrm{V} \cdot \mathrm{s}$ at $5 \mathrm{mTorr}$. It increases with the increase in Ar gas pressure up to 15 mTorr.

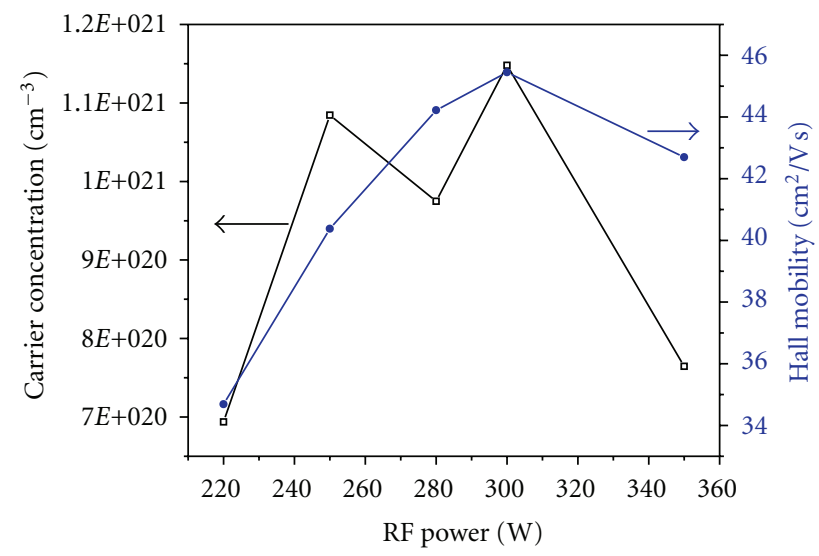

FIGURE 7: Carrier concentration and hall mobility with variations of RF power.

It reaches the maximum value of $45.5 \mathrm{~cm}^{2} / \mathrm{V} \cdot \mathrm{s}$ and thereafter decreases slightly to $40.5 \mathrm{~cm}^{2} / \mathrm{V} \cdot \mathrm{s}$ at 20 mTorr. A similar behavior was also observed in the carrier concentration of the films. The mobility and carrier concentration variations are directly related to the observed characteristics of electrical resistivity, that is, they are governed by the stoichiometric variation of the film. Even though the ITiO films in this work had the strong peak of (222) plane as well as the 


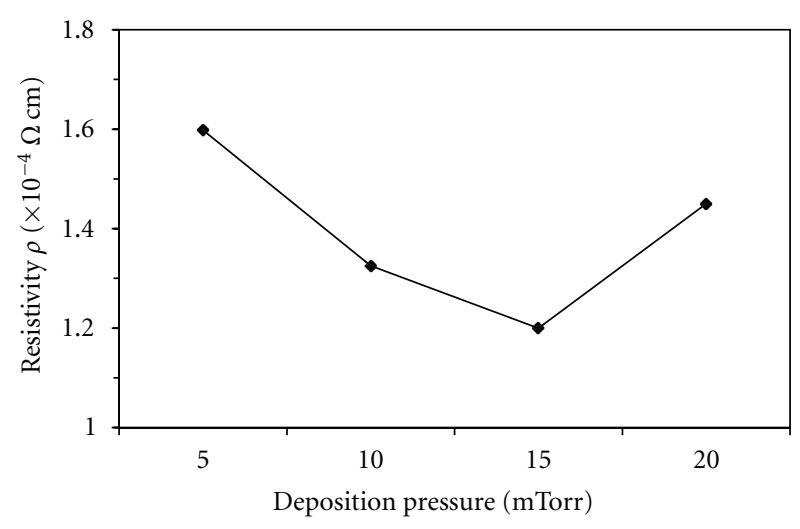

FIGURE 8: Resistivity of the ITiO film with variations of Ar gas pressure.

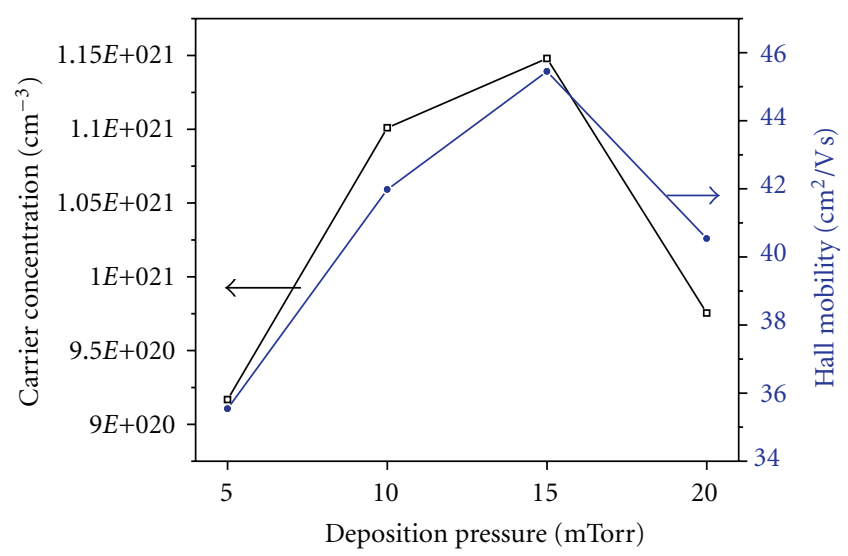

FIGURE 9: Carrier concentration and hall mobility with variations of Ar gas pressure.

very weak peaks of (400) and (440) crystal planes, they were found to be in good agreement with crystalline indium oxide. No extra peaks of impurity phases due to the addition of titanium in the indium oxide films were observed in Figures 1 and 2 of XRD results. This means that the ITiO film deposited by RF magnetron sputtering method is well crystallized without the presence of metallic $\mathrm{Ti}$ or $\mathrm{TiO}$ metal oxide phases under the appropriate conditions of sputtering power and gas pressure. Moreover the correlation between electrical resistivity and hall mobility could be explained by the morphological properties of the film with varying the gas pressure. From the analysis of AFM morphology in Figure 5, it was confirmed that the crystalline grains could not grow at lower gas pressures and the porosity was observable on the surface. This means that the grain size is small and the grain boundaries increase, thus decreasing hall mobility at lower gas pressures than $15 \mathrm{mTorr}$. As a consequence, the reduced hall mobility with increasing grain boundaries at lower gas pressure leads to bad electrical conduction. As far as the gas pressure is concerned, the mean free path of electron in the glow discharge plasma affects the kinetic energy of sputtered atom. As discussed earlier, at higher gas pressure over $15 \mathrm{mTorr}$, the energetic and mobile ITiO molecules cannot be sputtered effectively because the electrons have shorter mean free path, and they cannot have enough energy to ionize Ar gas atoms. Consequently the reduced mobility and carrier concentration due to the poorer morphology at higher gas pressure over $15 \mathrm{mTorr}$ produce higher electrical resistivity.

The optical transmittance also depends on the sputtering power and gas pressure. The average optical transmittance of $75 \%$ was obtained in the wavelength range of the visible spectrum. The highest value of $80 \%$ was obtained for the film deposited at $300 \mathrm{~W}$ of discharge power and $15 \mathrm{mTorr}$ of gas pressure.

\section{Conclusions}

In this paper, the titanium-doped indium oxide (ITiO) films under various deposition conditions of gas pressure and $\mathrm{RF}$ power were deposited on glass substrate by using RF magnetron sputtering method, with ITiO target composited of $97.5 \mathrm{wt} \% \mathrm{In}_{2} \mathrm{O}_{3}$ and $2.5 \mathrm{wt} \% \mathrm{TiO}_{2}$. Based on the experiments, the optimal deposition conditions of ITiO film of RF power and gas pressure were $300 \mathrm{~W}$ and $15 \mathrm{mTorr}$, respectively. The film showed the strongest XRD peak and the lowest volume resistivity under these conditions. An increment in sputtering power promotes the growth of crystalline and induces an improvement in electrical resistivity of the film. However, the excessive supply of the sputtering power over than $300 \mathrm{~W}$ may cause a degradation of the preferred orientation and limits the growth of crystalline grain. The morphological and electrical resistivity dependencies of the film on the gas pressure can be understood by the analysis of hall mobility and carrier concentration. The lowest volume resistivity was recorded as $1.2 \times 10^{-4} \Omega$-cm, and this value is sufficiently low comparable with that of conventional ITO or $\mathrm{ZnO}: \mathrm{Al}$ transparent conducting layer. The average optical transmittance was around $75 \%$ in the wavelength range of the visible spectrum.

\section{Acknowledgment}

This research was supported by Kyungsung University Research Grants in 2012.

\section{References}

[1] L. T. Yan and R. E. I. Schropp, "Changes in the structural and electrical properties of vacuum post-annealed tungstenand titanium-doped indium oxide films deposited by radio frequency magnetron sputtering," Thin Solid Films, vol. 520, no. 6, pp. 2096-2101, 2012.

[2] A. E. Delahoy, L. Chen, M. Akhtar, B. Sang, and S. Guo, "New technologies for CIGS photovoltaics," Solar Energy, vol. 77, no. 6, pp. 785-793, 2004.

[3] C. H. Yang, S. C. Lee, T. C. Lin, and W. Y. Zhuang, "Optoelectronic properties of titanium-doped indium-tin-oxide films deposited by RF magnetron sputtering at room temperature," Materials Science and Engineering B, vol. 134, no. 1, pp. 68-75, 2006.

[4] Y. Abe and N. Ishiyama, "Titanium-doped indium oxide films prepared by d.c. magnetron sputtering using ceramic target," 
Journal of Materials Science, vol. 41, no. 22, pp. 7580-7584, 2006.

[5] J. W. Bowers, H. M. Upadhyaya, T. Nakada, and A. N. Tiwari, "Effects of surface treatments on high mobility ITiO coated glass substrates for dye sensitized solar cells and their tandem solar cell applications," Solar Energy Materials and Solar Cells, vol. 94, no. 4, pp. 691-696, 2010.

[6] D. W. Han, J. Darma, E. Kuantama, D. J. Kwak, and Y. M. Sung, "Titanium-doped indium oxide films prepared by RF magnetron sputtering," in Proceedings of the 23rd International Symposium on Discharges and Electrical Insulation in Vacuum (ISDEIV'08), pp. 599-602, September 2008.

[7] J. H. Heo, K. Y. Jung, D. J. Kwak, D. K. Lee, and Y. M. Sung, "Fabrication of titanium-doped indium oxide films for dyesensitized solar cell application using reactive RF magnetron sputter method," IEEE Transactions on Plasma Science, vol. 37, no. 8, pp. 1586-1592, 2009.

[8] D. J. Kwak, M. W. Park, and Y. M. Sung, "Discharge power dependence of structural and electrical properties of Al-doped $\mathrm{ZnO}$ conducting film by magnetron sputtering (for PDP)," Vacuum, vol. 83, no. 1, pp. 113-118, 2008.

[9] L. T. Yan, J. K. Rath, and R. E. I. Schropp, "Electrical properties of vacuum-annealed titanium-doped indium oxide films," Applied Surface Science, vol. 257, no. 22, pp. 9461-9465, 2011.

[10] Z. Song, Q. Fu, L. Li, L. Li, Y. An, and Y. Wang, "Ti-doped $\mathrm{In}_{2} \mathrm{O}_{3}$ transparent conductive thin films with high transmittance and low resistivity," Optica Applicata, vol. 40, no. 4, pp. 751-757, 2010. 

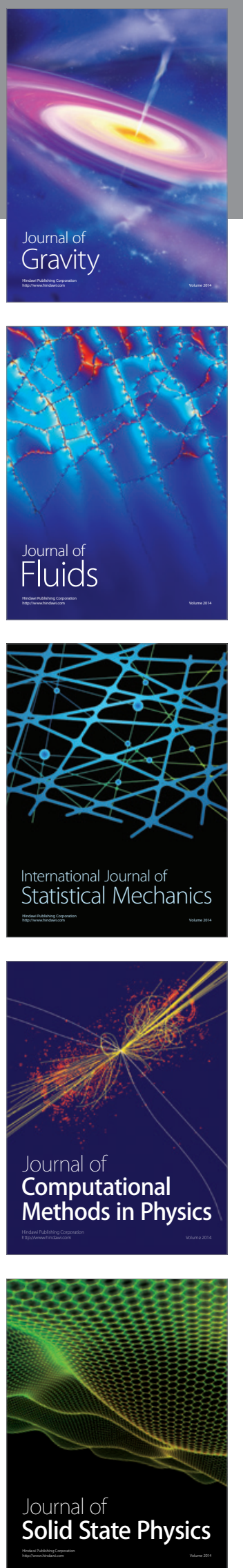

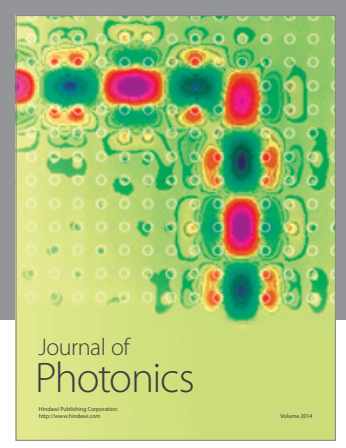

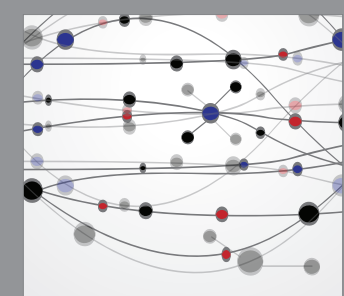

The Scientific World Journal
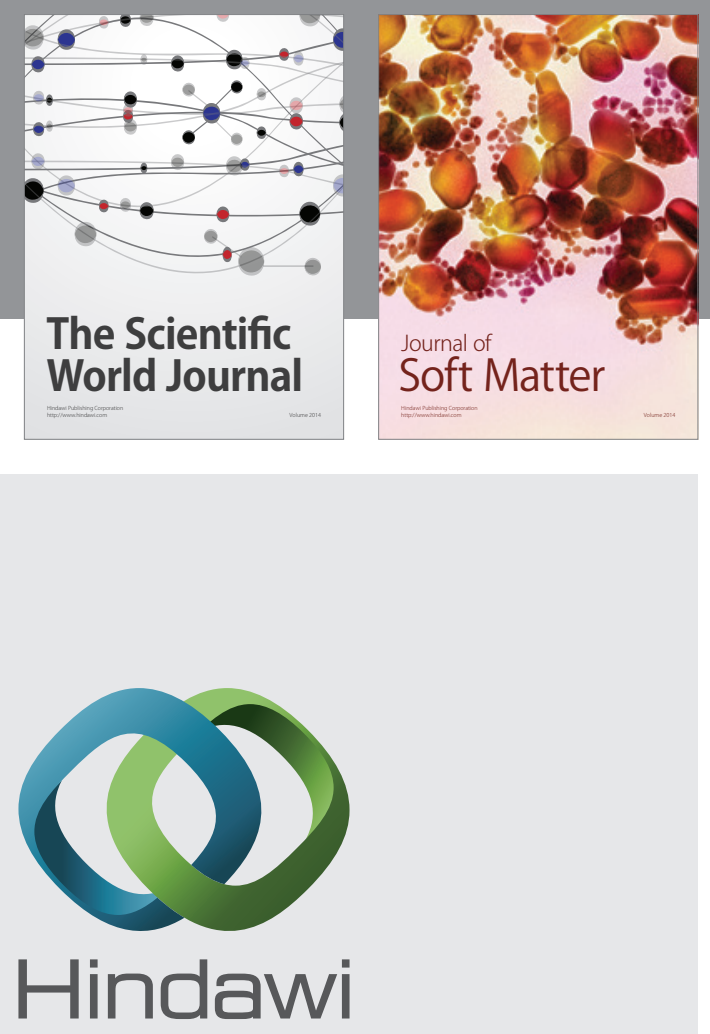

Submit your manuscripts at

http://www.hindawi.com
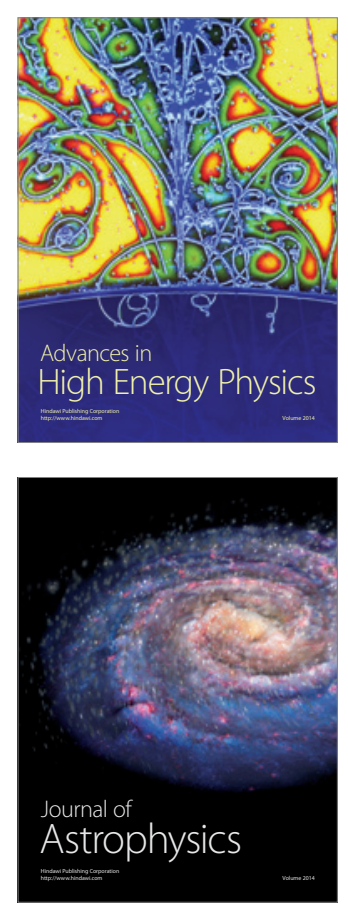
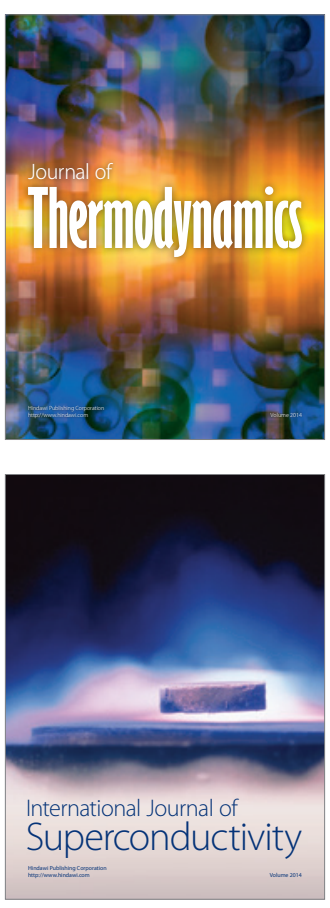
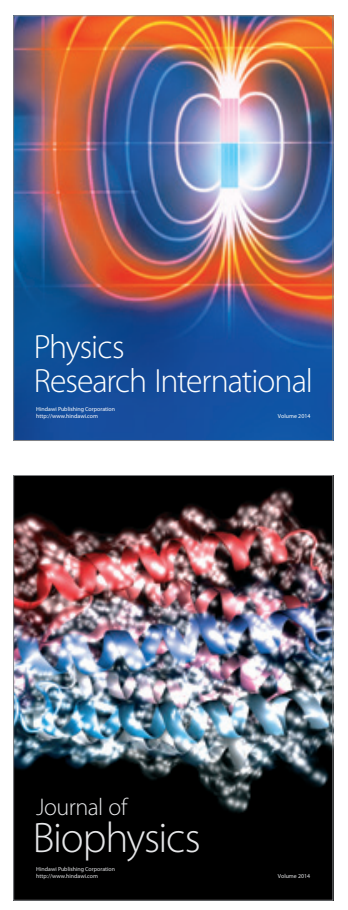
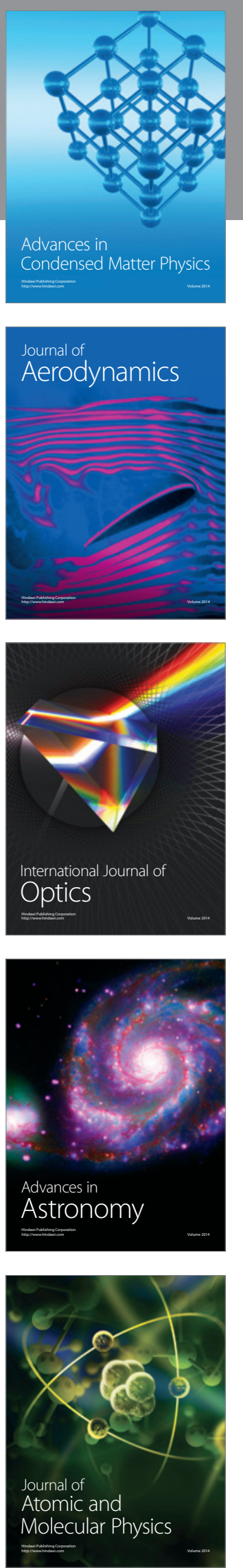